\title{
The Influence of Nonpharmacological Complex Therapy Conducted at a Community Day-Care Center on Cognitive function and Mood in Older Adults
}

This article was published in the following Dove Press journal:

Risk Management and Healthcare Policy

\author{
Agnieszka Gorzkowska (iD) \\ Izabela Zacharska- \\ Quaium (iD ${ }^{2}$ \\ Joanna Cholewa (D) ${ }^{3}$ \\ Jarosław Cholewa (iD) ${ }^{4}$
}

'Department of Neurorehabilitation, Faculty of Medical Sciences in Katowice, Medical University of Silesia, Katowice, Poland; ${ }^{2}$ Community Day-Care Center by Chance for Health Association in GolubDobrzyn, District Hospital, GolubDobrzyn, Poland; ${ }^{3}$ Department of Physical Education and Adapted Physical Activity, Institute of Physiotherapy and Health Sciences, The Jerzy Kukuczka Academy of Physical Education in Katowice, Katowice, Poland; ${ }^{4}$ Department of Health Related Physical Activity and Tourism, The Jerzy Kukuczka Academy of Physical Education in Katowice, Katowice, Poland
Correspondence: Jarosław Cholewa Department of Health Related Physical Activity and Tourism, The Jerzy Kukuczka Academy of Physical Education in Katowice, Mikołowska 72A str, Katowice 40-065, Poland

Tel +48 60I5300II

Fax +48338542166

Email j.cholewa@awf.katowice.pl
Introduction: Nonpharmacological interventions in the elderly may lead to the reduction of cognitive and depressive symptoms. The aim of the study was to evaluate changes in cognitive functions and mood in older adults participating in therapy, conducted in the community day-care center (CD-CC).

Patients and Methods: The study group (SG) included 46 elderly adults (21 M, $25 \mathrm{~W})$, the control group (CG) included 45 adults $(12 \mathrm{M}, 33 \mathrm{~W})$, who participated in the activities of the University of the Third Age. The following measuring tools were used: Mini-Mental State Examination, Clock-Drawing Test, Verbal Fluency Test, Digit Span Test, Stroop Color and Word Test, Beck's Depression Inventory, and Hospital Anxiety and Depression Scale. The intervention consisted of CD-CC 6-month nonpharmacological therapy.

Results: In the $\mathrm{SG}$, compared to the $\mathrm{CG}$, the scores on all the cognitive tests were significantly lower, Beck’s Depression Inventory was significantly higher. After intervention, the SG and the CG did not show substantial differences in their scores on the Mini-Mental State Examination, Clock-Drawing Test, and Beck's Depression Inventory. In the SG, a significant improvement was reported on the Verbal Fluency Test, Beck's Depression Inventory and Hospital Anxiety and Depression Scale scores.

Conclusion: The CD-CC complex therapy can be helpful for cognitive and emotional elderly functioning.

Keywords: cognition, depression, aging, elderly, community day-care center

\section{Introduction}

Optimization of the care for older adults, with mental disorders, and in particular with chronically disturbed mental functioning of organic origin, related to comorbidities associated with the aging process, represents an enormous challenge for all of the healthcare systems in the developed countries, worldwide. ${ }^{1,2}$

The fact that elderly age consists of an increasingly prolonged stage of the human life, causing the occurrence of biological processes naturally associated with advanced age, has become a growing challenge that inspires great interest among researchers, partially because the results of many studies on aging can determine, to some degree, current political and socio-economic decisions on a national scale.

The most common mental disorders that occur in our society include depression and mood disorders, such as sadness, anxiety, and fear. ${ }^{3}$ Prevalence of depressive 
disorders in elderly persons is estimated at approximately $4-6 \%$ of the population, and among patients of primary care physicians, it is even higher (about $9-15 \%$ ). ${ }^{4}$ Cognitive decline is also considered one of the most frightening aspects of aging. ${ }^{5}$ The prevalence of cognitive impairment increases with age.

Community day-care centers (CD-CCs) (also known as self-help CD-CCs) are social service institutions that have functioned in Poland since 1995. CD-CCs type C are designated for individuals with so-called other chronic disturbances of mental functions, including elderly persons who suffer from chronic mental disorders due to underlying organic causes, relevant to comorbidities associated with advancing age. A CD-CC facility provides services at least 5 days a week, for 8 hours daily, including at least 6 hours of therapy per day. In many countries, similar forms of systematic therapeutic programs, including mostly occupational therapy for elderly persons have been designed and implemented (eg, in Sweden or the USA). The low-intensity psychological interventions (self-help, psychoeducation, bibliotherapy, internet cognitivebehavioral therapy: iCBT) for older adults with mild-tomoderate mental health problems can be effective. ${ }^{6}$

Nonpharmacological treatment options for the treatment of older people's problems have been dynamically developed in the last few years. Patients with cognitive impairment and depression might benefit from ongoing and collaborative working between pharmacological and non-pharmacological treatments, including repetitive transcranial magnetic stimulation (rTMS), transcranial direct current stimulation (tDCS), cognitive training, exercise and non-exercise physical activities, complementary and alternative medicine such as Shiatzu. ${ }^{7-9}$ In this context, many mechanisms have been explored. Recently, it has been proposed that a benefit mechanism of action of noninvasive brain stimulation techniques in dementia may be the modulation of neurotrophin release, although systematic studies in humans are still lacking. ${ }^{10}$

Attempts at quality improvement in community mental health services for older people are stymied by a lack of robust measures. ${ }^{11}$

CD-CC represents a place, in which a nonpharmacological therapy for groups of elderly persons is conducted. For this reason, it appears to be one of the best structures that can create favorable conditions for social integration and general activation of older persons in their local community (while staying in their own homes does not cause the discomfort of institutionalization). ${ }^{12}$ For this reason, $\mathrm{CD}-\mathrm{CC}$ could be a desirable environment for research purposes.

The aim of this study was to evaluate the influence of nonpharmacological complex therapy conducted for 6 months in the CD-CC on changes in cognitive functions and mood among the elderly participants.

\section{Patients and Methods}

A total of 91 persons were enrolled into the study. All patients provided written informed consent for their details to be used in this report.

The study group (SG) included 46 elderly adults, 21 men (M) and 25 women (W) with mental disorders, due to organic origin, related to morbidities associated with aging, who attended $\mathrm{CD}-\mathrm{CC}$. The inclusion criteria to the SG were as follows: 1) a recommendation for obtaining services at the CD-CC type $\mathrm{C}$, meaning the other chronic mental disorders, including among others, chronic disturbances of mental functioning of organic origin related to comorbidities associated with aging; 2) an ability to understand the study goal and to perform psychological tests; 3 ) age $\geq 55$ years; and 4) a signed informed consent form. The main exclusion criteria were serious somatic and mental diseases (other than in point 1 of the inclusion criteria), which may have a substantial influence on cognitive and physical level of functioning.

The control group (CG) included 45 adults, aged $\geq$ 55 years $(12 \mathrm{M}$ and $33 \mathrm{~W})$, who participated in the activities of the University of the Third Age (U3A). The inclusion criteria to the $C G$ were as follows: 1) absence of serious somatic and mental diseases; 2) an ability to understand the study goal and to perform neuropsychological tests; 3 ) age $\geq 55$ years; and 4 ) a signed informed consent form.

The following methodological tools of appraisal were used in this study: Survey of demographic data, MiniMental State Examination (MMSE), Clock-Drawing Test (CDT), Verbal Fluency Test (VFT), Digit Span Test (DST), Stroop Color and Word Test (SCWT), Beck's Depression Inventory (BDI), and Hospital Anxiety and Depression Scale (HADS). The selection of neuropsychological tests used resulted from a literature review and the authors' experience. Tests were selected from those for which age sensitivity was confirmed in the subject literature. Two simple screening tests (MMSE and CDT) were combined in this work, as in practice, to enhance their diagnostic value. The assessment of verbal fluency provides important information and seems to be more sensitive to the features of cognitive disorders in pathologically aging people. The 
authors show a decrease in VFT in the elderly in many studies. ${ }^{13}$ In gerontological neuropsychology, the presence of executive problems identified by the SCWT, such as impairment of control mechanisms, planning problems and suppression of adverse associations is indicated in the aging process. The DST performance has been found to be influenced by demographic factors and the relationship between older age and reduced DST performance has been consistently reported. ${ }^{14}$ Neuropsychological assessments were conducted by an experienced psychologist, one patient was examined by one psychologist at all stages.

A study intervention in the SG consisted of 6-month nonpharmacological actions (complex therapy) conducted at the CD-CC (Table 1).

An evaluation of the cognitive and emotional functioning in the SG conducted at the study initiation (baseline), and after 6-month nonpharmacological actions conducted at the CD-CC.

Table I Weekly Task Plan and Schedule of the Therapy Conducted at the CD-CC

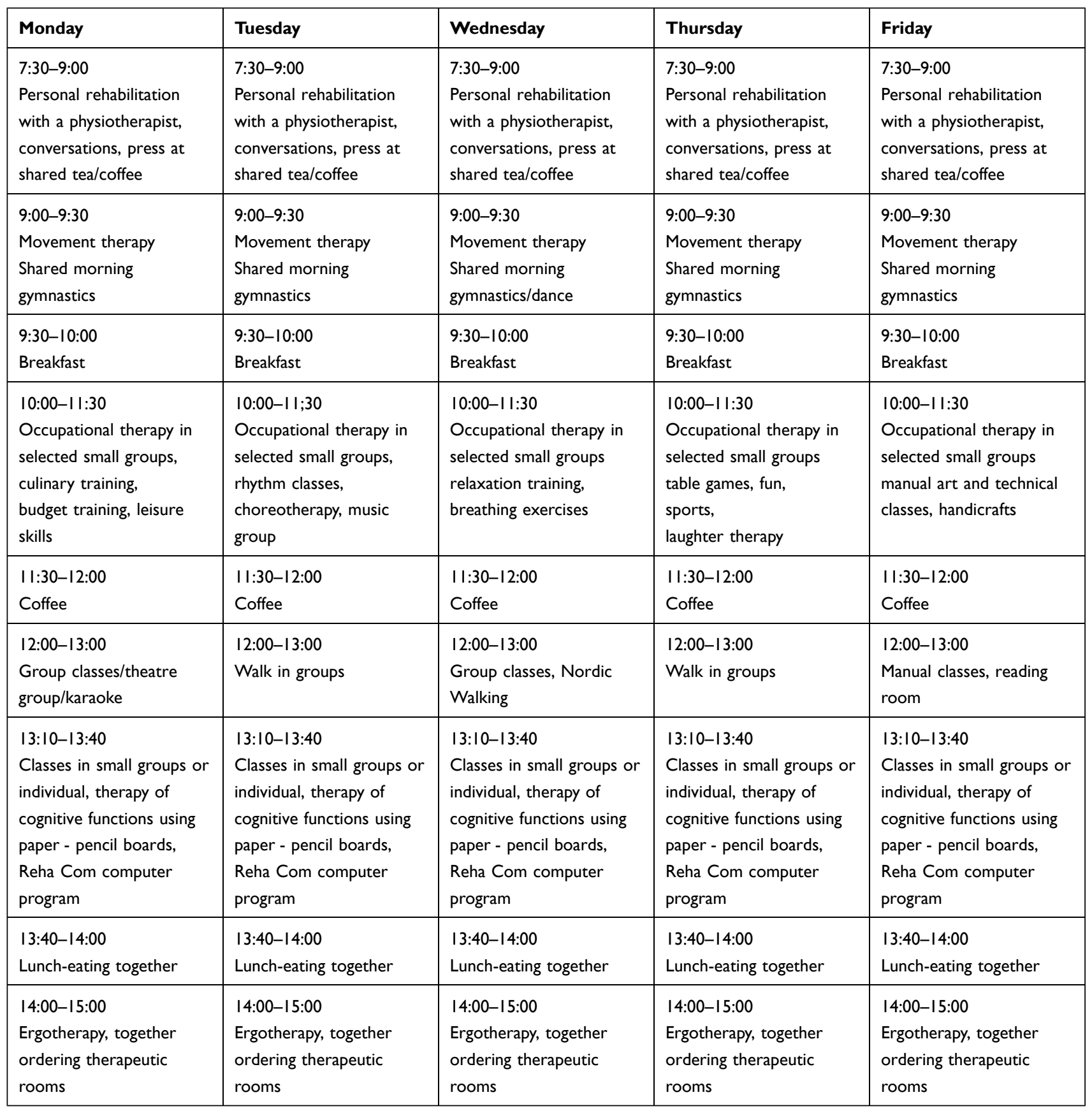


Table 2 Characteristics of the Study and Control Groups

\begin{tabular}{|c|c|c|c|}
\hline Variables & $\begin{array}{l}\text { SG } \\
n=46\end{array}$ & $\begin{array}{l}\text { CG } \\
n=45\end{array}$ & p-value* \\
\hline Sex: women/men [\%] & $25 / 21$ & $33 / 12$ & NS \\
\hline Age [years] & 66 [range: 56-85] & 69 [range 58-86] & NS \\
\hline $\begin{array}{l}\text { Place of residence } \\
\text { town/village [\%] }\end{array}$ & $9 / 91$ & $67 / 33$ & $p<0.001$ \\
\hline $\begin{array}{l}\text { Type of residence } \\
\text { single/with family [\%] }\end{array}$ & $50 / 50$ & $47 / 53$ & NS \\
\hline $\begin{array}{l}\text { Assessment of material situation } \\
\text { good/medium/bad [\%] }\end{array}$ & $17 / 74 / 9$ & $35 / 59 / 6$ & NS \\
\hline $\begin{array}{l}\text { Education } \\
\text { higher/vocational/secondary/primary [\%] }\end{array}$ & $0 / 25 / 11 / 64$ & $13 / 2742 / 18$ & $p=0.001$ \\
\hline $\begin{array}{l}\text { Functional efficiency } \\
\text { independent/needs help [\%] }\end{array}$ & $94 / 6$ & $100 / 0$ & NS \\
\hline $\begin{array}{l}\text { Other mental disorders [\%]: } \\
\text { Depression } \\
\text { Anxiety disorders } \\
\text { Affective disorders } \\
\text { Mood and personality disorders } \\
\text { Dementia }\end{array}$ & $\begin{array}{l}40 \\
7 \\
6 \\
17 \\
30\end{array}$ & - & - \\
\hline
\end{tabular}

Notes: ${ }^{*}$ Chi-square test, $p<0,05$.

A statistical analysis was conducted by using the Statistica 12.0 (Dell Software Information Management Group) program. In all of the tests, the $95 \%$ confidence level was used, and $p<0,05$ was considered as statistically significant. Characteristics of the SG and CG are presented in Table 2.

A comparison of the cognitive test results between the SG and the CG, at baseline (before starting the study intervention for the SG) is presented in Table 3.

\section{Results}

The cognitive tests results in the $\mathrm{CG}$ remained within normal limits, while in the SG they were indicative of mild cognitive impairment. Considering, according to the literature, the cut-off value for MMSE as below 24 points, it was found that in 24 participants $(52 \%)$ in the $\mathrm{SG}$, clinical symptoms of dementia were present, while in the $\mathrm{CG}$, clinical symptoms of dementia were present in only 9 participants (20\%). MMSE score was standard adjusted for education level and age. Considering, according to the literature, the cut-off value for CDT as above 1 point, it was found that in 21 participants $(46 \%)$ in the $\mathrm{SG}$, clinical symptoms of dementia were present, while in the $\mathrm{CG}$, clinical symptoms of dementia were present in only 5 participants (11\%).

A comparison of the cognitive test results in the SG between the baseline (before starting the study) and after 6-month therapy intervention is presented in Table 4.

After the therapeutic nonpharmacological intervention conducted at the $\mathrm{CD}-\mathrm{CC}$, in the SG the number of participants in whom cognitive impairment at the level of dementia (based on the MMSE scores) had been found, was decreased from $24(52 \%)$ to 20 participants (43\%). In the CG, mild cognitive impairment symptoms were reported in 9 participants $(20 \%)$. Similarly, in the CDT, in the SG, the number of participants with the scores indicative of dementive disorders, was decreased from 21 participants (46\%) before the therapy to 14 participants $(30 \%)$ after the therapy. In the $\mathrm{CG}$, based on the CDT, dementia symptoms was found in 5 participants (11\%). A comparison of the cognitive test results between the $\mathrm{SG}$ and $\mathrm{CG}$, after 6-month therapy intervention (in the $\mathrm{SG}$ ) is presented in Table 5.

A comparison of the BDI and HADS test results between the SG and the CG at baseline (before the study intervention in the SG) is presented in Table 6. 
Table 3 Cognitive Tests Results in Groups Before Intervention

\begin{tabular}{|l|l|l|l|l|l|}
\hline \multirow{2}{*}{ Test } & \multicolumn{2}{l|}{ SG } & \multicolumn{2}{l|}{ CG } & \multirow{2}{*}{$*^{*}$} \\
\cline { 2 - 5 } & $\mathbf{M}$ & SD & M & SD & \\
\hline MMSE & 23.174 & 4.963 & 26.267 & 3.033 & 0.002 \\
CDT & 1.5 & $0,8 I$ & 1.133 & 0.405 & 0.012 \\
L-VFT correctly & 10.594 & 2.608 & 11.778 & 3.269 & 0.105 \\
L-VFT errors & 1.789 & 1.408 & 1.037 & 1.427 & 0.003 \\
C-VFT correctly & 12.587 & 5.256 & 12.600 & 6.390 & 0.673 \\
C-VFT errors & 0.435 & 0.75 & 0.200 & 0.548 & 0.198 \\
DST F & 7.108 & 1.16 & 7.178 & 2.863 & 0.445 \\
DST B & 4.304 & 9.138 & 4.156 & 2.374 & 0.023 \\
DST T & 9.886 & 3.366 & 11.267 & 4.779 & 0.047 \\
SCWT I time & 47.104 & 35.829 & 40.758 & 36.368 & 0.020 \\
SCWT II time & 50.867 & 41.217 & 48.444 & 45.639 & 0.044 \\
SCWT I errors & 0.261 & 0.743 & 0.111 & 0.611 & 0.387 \\
SCWT II errors & 1.000 & 2.459 & 1.444 & 3.671 & 0.450 \\
\hline
\end{tabular}

Note: *Test U Mann-Whitney.

Abbreviations: MMSE, Mini Mental State Examination; CDT, Clock Drawing Test; VFT, Verbal Fluency Test; Letter VFT; Category VFT (Animals); DST, Digit Span Test; DST Forward; DST Backward; DST TOTAL; SCWT, Stroop Color and Word Test; SCWT part I; SCWT part II; M, arithmetic average; SD, standard deviations.

Table 4 Cognitive Tests' Results in the Study Group Before and After Intervention

\begin{tabular}{|l|l|l|l|l|l|}
\hline \multirow{2}{*}{ Test } & \multicolumn{2}{l|}{$\begin{array}{l}\text { Before } \\
\text { Intervention }\end{array}$} & \multicolumn{2}{l|}{$\begin{array}{l}\text { After } \\
\text { Intervention }\end{array}$} \\
\cline { 2 - 5 } & $\mathbf{M}$ & SD & M & SD & \\
\hline MMSE & 23.174 & 4.963 & 23.913 & 5.299 & 0.065 \\
CDT & 1.5 & $0.8 I$ & 1.391 & 0.856 & 0.372 \\
L-VFT correctly & 10.594 & 2.608 & 10.985 & 2.649 & 0.118 \\
L-VFT errors & 1.789 & 1.408 & 1.079 & 1.180 & 0.001 \\
C-VFT correctly & 12.587 & 5.256 & 12.717 & 6.742 & 0.941 \\
C-VFT errors & 0.435 & 0.75 & 0.261 & 0.535 & 0.263 \\
DST F & 7.1080 & 1.816 & 6.956 & 2.118 & 0.727 \\
DST B & 4.304 & 9.138 & 2.652 & 1.864 & 0.457 \\
DST T & 9.886 & 3.366 & 9.608 & 3.276 & 0.229 \\
SCWT I time & 47.104 & 35.829 & 44.460 & 39.616 & 0.472 \\
SCWT II time & 50.867 & 41.217 & 47.223 & 26.287 & 0.933 \\
SCWT I errors & 0.261 & 0.743 & 0.087 & 0.285 & 0.123 \\
SCWT II errors & 1.000 & 2.459 & 0.609 & 1.000 & 0.39 \\
\hline
\end{tabular}

Note: *Wilcoxon pairs order test.

Abbreviations: MMSE, Mini Mental State Examination; CDT, Clock Drawing Test; VFT, Verbal Fluency Test; Letter VFT; Category VFT (Animals); DST, Digit Span Test; DST Forward; DST Backward; DST TOTAL; SCWT, Stroop Color and Word Test; SCWT part I; SCWT part II.

Considering, according to the literature, the cut-off value for BDI as $>10$ points, it was found that in 29 participants $(63 \%)$ in the SG, clinical symptoms of depression were present, while in the CG, clinical symptoms of depression were present in 19 participants $(42 \%)$.
Table 5 Cognitive Tests' Results in Study and Control Groups After Intervention

\begin{tabular}{|l|l|l|l|l|l|}
\hline \multirow{2}{*}{ Test } & \multicolumn{3}{l|}{ SG } & \multicolumn{2}{l|}{ CG } \\
\cline { 2 - 5 } & M & SD & M & SD & \\
\hline MMSE & 23.913 & 5.299 & 26.267 & 3.033 & 0.103 \\
CDT & 1.391 & 0.856 & 1.133 & 0.405 & 0.405 \\
L-VFT correctly & 10.986 & 2.649 & 3.269 & 3.269 & 0.306 \\
L-VFT errors & 1.080 & 1.180 & 1.427 & 1.427 & 0.545 \\
C-VFT correctly & 12.717 & 6.742 & 6.390 & 6.390 & 0.915 \\
C-VFT errors & 0.261 & 0.535 & 0.548 & 0.548 & 0.610 \\
DST F & 6.957 & 2.118 & 2.863 & 2.863 & 0.284 \\
DST B & 2.652 & 1.864 & 2.374 & 2.374 & 0.003 \\
DST T & 9.609 & 3.276 & 4.779 & 4.779 & 0.019 \\
SCWT I time & 44.46 & 39.616 & 40.758 & 36.368 & 0.057 \\
SCWT II time & 47.223 & 26.287 & 48.444 & 45.639 & 0.034 \\
SCWT I errors & 0.087 & 0.285 & 0.111 & 0.611 & 0.743 \\
SCWT II errors & 0.609 & 1.000 & 1.444 & 3.671 & 0.499 \\
\hline
\end{tabular}

Note: *U Mann-Whitney test.

Abbreviations: MMSE, Mini Mental State Examination; CDT, Clock Drawing Test; VFT, Verbal Fluency Test; Letter VFT; Category-VFT (Animals); DST, Digit Span Test; DST Forward; DST Backward; DST TOTAL; SCWT, Stroop Color and Word Test; SCWT part I; SCWT part II.

Table $6 \mathrm{BDI}$ and HADS Results in Both Groups Before Intervention

\begin{tabular}{|l|l|l|l|l|l|}
\hline \multirow{2}{*}{ Test } & \multicolumn{2}{|l|}{ SG } & \multicolumn{2}{l|}{ CG } & \multirow{2}{*}{ * } \\
\cline { 2 - 5 } & M & SD & M & SD & \\
\hline BDI & 14.087 & 7.947 & 11.133 & 9.445 & 0.014 \\
HADS D & 7.326 & 3.700 & 5.867 & 3.823 & 0.052 \\
HADS L & 5.217 & 4.027 & 3.978 & 3.876 & 0.075 \\
\hline
\end{tabular}

Note: *U Mann-Whitney test.

Abbreviations: BDI, Beck Depression Inventory; HADS, Hospital Anxiety and Depression Scale; HADS D, depression scale; HADS L, anxiety scale.

Table 7 BDI and HADS Results in Study Group Before and After Intervention

\begin{tabular}{|l|l|l|l|l|l|}
\hline \multirow{2}{*}{ Test } & \multicolumn{2}{|l|}{$\begin{array}{l}\text { Before } \\
\text { Intervention }\end{array}$} & \multicolumn{2}{l|}{$\begin{array}{l}\text { After } \\
\text { Intervention }\end{array}$} & \\
\cline { 2 - 5 } & $\mathbf{M}$ & SD & $\mathbf{M}$ & SD & \\
\hline BDI & 13.392 & 7.993 & 8.882 & 7.016 & 0.00002 \\
HADS D & 7.02 & 3.744 & 4.02 & 3.717 & 0.00001 \\
HADS L & 5.216 & 3.997 & 3.196 & 3.188 & 0.00056 \\
\hline
\end{tabular}

Note: *Wilcoxon pairs order test.

Abbreviations: BDI, Beck Depression Inventory; HADS, Hospital Anxiety and Depression Scale; HADS D, depression scale; HADS L, anxiety scale.

Upon applying the HADS test, using a universally accepted cut-off value $>7$ points, the clinical symptoms of depression were found in the SG in 10 participants (21\%), and in the $\mathrm{CG}$, in 8 participants $(17 \%)$. 
Table $8 \mathrm{BDI}$ and HADS Results in Study and Control Groups After Intervention

\begin{tabular}{|l|l|l|l|l|l|}
\hline \multirow{2}{*}{ Test } & \multicolumn{2}{l|}{ SG } & \multicolumn{2}{l|}{ CG } & \multirow{2}{*}{$*$} \\
\cline { 2 - 5 } & M & SD & M & SD & \\
\hline BDI & 9.326 & 7.171 & 11.133 & 9.445 & 0.638 \\
HADS D & 4.239 & 3.766 & 5.867 & 3.823 & 0.024 \\
HADS L & 3.065 & 3.094 & 3.978 & 3.876 & 0.349 \\
\hline
\end{tabular}

Note: *U Mann-Whitney test.

Abbreviations: BDI, Beck Depression Inventory; HADS, Hospital Anxiety and Depression Scale; HADS D, depression scale; HADS L, anxiety scale.

Subsequently, symptoms of anxiety were present in 25 participants $(54 \%)$ in the $\mathrm{SG}$, and in 14 participants $(31 \%)$ in the CG. A comparison of the BDI and HADS test results in the SG, between the baseline (before the study intervention) and after the study intervention is presented in Table 7.

The mean scores in the SG before the study intervention were in the range of values for depressive disorder, according to both the BDI and the HADS scales. In contrast, after the study intervention, the scores were within normal range. A comparison of the BDI and HADS test results between the SG (after the study intervention) and the CG is presented in Table 8 . In addition, after the study intervention, no evidence of depressive or anxiety disorders was found in the SG, and the symptoms of depression, based on the HADS scale, had significantly lower intensity than in the CG.

\section{Discussion}

\section{Cognitive Functioning}

Elderly persons admitted to the community day-care center (CD-CC) type $\mathrm{C}$ display significantly worse cognitive functioning level, compared to a general older population. Observations made in our study confirms that cognitive aging patterns are individualized and very diverse (they fall within the broad spectrum of physiology and pathology). This may be due to the fact that individual brain structures age at different rates, but also because the hypothetical individual brain reserve somehow counteracts neuropathological processes that impair the mental performance of the elderly. In our study, we report that the participants from the SG scored worse in almost all of the tests evaluating cognitive performance (that were used in the study), compared to the participants from the CG. In fact, these results were expected, because of the indications for admission to the CD-CC, which include cognitive dysfunctions and disorders, both primary and secondary, due to associated somatic and mental diseases.
It should be highlighted that all the applied tests have revealed a good sensitivity in the range of identification of cognitive function abnormalities among the elderly persons, and were able to distinguish between the SG and the CG. This confirms the usefulness of the applied measuring tools in the study population, and indicates a selection of a short set of screening tests for evaluation of elderly persons admitted to the CD-CC (based on those, which were used in our study). The obtained results have also revealed a relatively general character of the identified disorders that can be possibly explained by frequently occurring depression (that was present in a large percentage in the SG) and attention disorders, which affect the performance level of all the remaining tests.

Simultaneously, it is interesting that despite obtaining statistically significant differences in the results from both groups, the absolute differences in these results were not very big. Therefore, we can presume that the properly adjusted intervention for elderly persons with chronic mental disorders (eg, chronic disturbances of mental functioning of the organic origin, related to comorbidities associated with aging) may improve their functioning to some degree, so that, they can achieve a similar level of the functional status to their healthy older peers. Based on the literature review, the expected improvement of mental functions can be achieved not only with regard to an intervention in the form of cognitive training, but also physical activity. For instance, in their study, Ji et al $(2018)^{15}$ found that physical exercises improved the walking speed and cognitive functions, involving some compensatory mechanisms (which activated both of these abilities, among the engaged patients). ${ }^{15}$ In our analysis of the effects of study intervention at the CD-CC, in the SG we have reported the improvement of the test scores compared to a baseline. In particular, in the SG, a statistically significant improvement after the 6-month intervention was obtained in the area of verbal fluency (measured by the VFT) that was manifested by a lower number of committed errors.

In the case of the remaining tests, the differences were not statistically significant. In contrast, it should be highlighted that after the 6-month intervention at the CD-CC, the results obtained in the $\mathrm{SG}$, in the majority of the examined areas were approximated to those from the CG. In contrast to the baseline scores, in the majority of tests, the differences between the SG and the CG (after the 6-month intervention in the SG) were not statistically significant. 
We can also treat the $\mathrm{CD}-\mathrm{CC}$ as some kind of social network. Researchers suggest that social networks modify the relation of some measures of Alzheimer's disease pathology in level of cognitive function. ${ }^{16}$ The lack of social interaction is associated with incident dementia. The strength of the associations between poor social interaction and incident dementia is comparable with other well-established risk factors for dementia, including low education attainment, physical inactivity, and late-life depression. ${ }^{17}$ Additionally Glei et $\mathrm{al}^{18}$ noted that participation in social activities outside the family (CD-CC is a form of these activities) may have a bigger impact on cognitive function than social contacts with family or nonrelatives. $^{18}$

This observation allows us to assume that the intervention conducted at the CD-CC had a positive impact on the cognitive functions in the elderly participants (with chronic disturbances of mental functioning of organic origin, related to comorbidities associated with aging). In the context of the studies described in the literature, relevant to the cognitive impairment in older age groups (particularly those afflicted by additional diseases and features of pathologic aging), the results of our study that have revealed some cognitive improvement, after the intervention in the SG, seem to be optimistic.

\section{Anxiety and Depression}

Progress in medicine, pharmacology, and improvement of living conditions in the contemporary world allow people to live longer, but their mental health deteriorates, due to coexisting somatic disorders, loss of independence and autonomy, feelings of being misunderstood, experience of injustice, lack of influence on the surroundings, loss of purpose in life, pessimism, and fear of new or unknown events and changes that in consequence can cause deteriorated mood and depression. ${ }^{19}$

In our study, elderly persons admitted to CD-CC type $\mathrm{C}$ had significantly worse results compared to the $\mathrm{CG}$ in the area of depression and anxiety symptoms measured by the BDI and HADS scales. The fact that the depressive symptoms were found in as many as $63 \%$ of persons qualified for the therapy at $\mathrm{CD}-\mathrm{CC}$ type $\mathrm{C}$ is not surprising, since this facility has a profile of the therapeutic center for persons with so-called other disorders of mental functions, such as depression. In contrast, $45 \%$ of persons with depressive features among the attendees of the U3A, confirms a very high prevalence of depression among elderly persons, even the active ones. The results of examination of depression in this population indicate that $10 \%$ of the patient population of family physicians suffer from depression. ${ }^{20}$ In our study, the percent of depression in older adults was 4.5 times higher. A possible cause of this phenomenon may be related to insufficient diagnosing of the family physicians' patient population. Therefore, it is currently indicated to perform a depression screening test in every elderly person.

In addition, in the SG, the anxiety symptoms were more prevalent - in 25 participants (54\%), compared to 14 participants (31\%) in the CG (based on the HADS scores). With the use of the HADS tool, the presence of clinical symptoms of depression was found in the SG in 10 participants $(21 \%)$, compared to 8 participants $(17 \%)$ in the CG. It should be noted that in the case of both of these measuring tools, the results are coherent and indicate a higher prevalence of depression in the SG, compared to the CG. In contrast, the different absolute values may be relevant to the various sensitivity of these scales in elderly adults attending CD-CC. However, at this point, the BDI appears to be a more accurate diagnostic tool.

It should be underscored that the intervention conducted at the CD-CC had a positive influence on the reduction of these symptoms among the study participants, who revealed on both scales statistically significant improvements. Both BDI and HADS have shown sensitivity in the area of assessment of mental functioning improvement, in older participants undergoing therapeutic intervention.

In an analysis of the SG scores, after the study intervention, it can be noted that according to the BDI scale and the anxiety HADS-A subscale, the proposed nonpharmacological intervention allowed us to approximate the mental functioning level in the SG and CG. An evaluation of the effects of nonpharmacological interventions (similar to the one that was implemented at the (D-CC) on the mental health status of elderly persons represents an important field of investigations for many researchers worldwide. ${ }^{21,22}$

A review of research on the impact of physical activity on mental and somatic status of elderly adults has indicated that the physical activity can be an alternative to pharmacological therapy for depressive disorders, and thus, it can affect the reduction of costs related to medical care for older persons. ${ }^{23-25}$ Furthermore, regular physical exercises, physical therapy, and respiratory exercises cause the reduction of depressive symptoms, mental disorders, and anxiety states. ${ }^{25}$ Moreover, the improvement occurs 
also in the area of social functioning, somatic conditions, and physical performance, as well as cognitive functions, attention span, and memory.

Interesting results were reported by Hirosaki et al $(2013)^{27}$ in a group of people over 60 years of age, who participated in a combined program of exercise and laughter therapy in Japan. This intervention has revealed benefits in both physical and mental health areas. In this study, it was found that positive emotions and laughter may contribute to an increase of motivation of elderly persons in the participation in exercises, and also can exert beneficial effects on the increase of glucose metabolism during physical activities. ${ }^{27}$ Similarly, in our study, the therapeutic interventions applied in the $\mathrm{CD}-\mathrm{CC}$, were also a source of positive emotions for the participants, and in consequence, they have been considered to be a factor of the mental status improvement.

In our study analysis of the therapeutic intervention conducted at the CD-CC, it should be pointed out that dance therapy represents a preferred form of activity among the study participants. Eyigor et al $(2009)^{28}$ have shown that dance therapy has a positive impact on physical performance, balance, and depressive symptom level, as well as the reduction of costs relevant to medical care for elderly persons. ${ }^{27}$ In this study, it has been revealed that after only 6 weeks of dance therapy, the intensity of depressive symptoms was decreased and physical performance of the older participants was increased. In particular, in the participants of this study, self-esteem, personality development, vitality sense, and inner equilibrium, as well as an ability to "reconnect" with bodily sensations and the quality of life were improved. ${ }^{28}$

Among the factors that contribute to cognitive decline in older adults, there is now agreement that depression in later life is one of the recognized clinical risk factors for dementia. $^{29}$ In patients in whom depression appears later in life the typical clinical presentations include psychomotor retardation, difficulty at work, apathy, lack of insight, and executive dysfunction. ${ }^{30}$

Moreover, many researchers believe that cognitive performance is related to the severity level of depression and in a consequence, together with the decrease of depressive symptoms, cognitive functions can often be improved. ${ }^{31,32}$ Such a relation has also been reported in our study, where these changes were parallel.

In the context of the improvement of emotional functioning in the SG, it can be assumed that the therapeutic interventions provided in the $\mathrm{CD}-\mathrm{CC}$ have positive influence on cognitive functions and mood in elderly persons attending such programs. Furthermore, these interventions may lead to the reduction of anxiety and depressive symptoms.

\section{Strengths and Limitations}

The topic of community day-care centers has rarely been studied in research. In this study, for the first time, we have tried to determine, in the standard fashion, the objective changes that occur in elderly persons using the therapeutic services in the CD-CC type $\mathrm{C}$.

Another advantage includes the application in this study was different from the previously used perspective of looking at the major tasks of CD-CC, transferring the traditional accent from a generally understood social integration, to which the applied therapy focused on concrete cognitive functions and aspects of emotional functioning (which may consist both the separate value and basis of improvement of the social integration).

The main limitations of our study include its pilot character and small sample size. Of course, upon conducting another observation in this area, the group size should be increased. In addition, we assessed how the therapy used naturally in the very heterogeneous group would work. In the future the recruited study participants should be stratified, and categorized to different groups, depending on their main diagnostic findings (in order to increase homogeneity within the groups). Moreover, it would be beneficial to implement a battery of neuropsychological tests that would reflect a slightly wider cognitive and emotional profile. Simultaneously, such assessments would consider both the larger impact of clinical and demographic parameters and the influence of the concrete type of provided intervention (eg, a specific type of occupational therapy) on the occurring changes (that are being examined).

The 6-month nonpharmacological CD-CC therapy used in the presented study was a natural procedure used in $\mathrm{CD}-\mathrm{CC}$ and was presented as far as it could be done in detail (Table 1), but it should be noted that detailed forms of therapy were not strictly required during our project and the specific form of therapy was selected by the therapist each time as happens in natural conditions. In the next study planned we will try to describe its parameters more specifically.

This study was not an RCT, but only a pre- and postintervention comparison. However, wanting to carry out a research project in the natural conditions of $\mathrm{CD}-\mathrm{CC}$, in order to evaluate the procedure applied naturally there, researchers have only this approach at their disposal or they will be deprived of the possibility of making an assessment. 
We treat our results as preliminary/pilot, and the final assessment of the effects of intervention in $\mathrm{CD}-\mathrm{CC}$ requires further research.

In future research projects it would also be important to consider an objective follow-up assessment that could facilitate evaluation of long-term effects of the specific therapy, both in persons who continue and terminate the utilization of supportive services at the CD-CC.

\section{Conclusion}

The nonpharmacological complex therapy rendered at the CD-CC can be helpful for the older adult population, in terms of the benefits in cognitive and emotional functioning. However, prospective studies on large populations are merited to further elucidate this topic.

\section{Ethics Statement}

The study was granted an approval by the Bioethical Commission of the University of Nicolaus Copernicus in Torun, Poland, at the Collegium Medicum of Ludwik Rydygier, in Bydgoszcz, Poland, (number of the approval KB 452/2016). The authors affirm that the guidelines outlined in the Declaration of Helsinki were met.

\section{Disclosure}

The authors report no conflicts of interest in this work.

\section{References}

1. Ferdosi M, Rezayatmand R, Molavi Taleghani Y. Risk management in executive levels of healthcare organizations: insights from a scoping review (2018). Risk Manag Healthc Policy. 2020;13:215-243. doi:10.2147/RMHP.S231712

2. Thammatacharee N, Suphanchaimat R. Long-term projections of patients undertaking renal replacement therapy under the universal coverage scheme in Thailand. Risk Manag Healthc Policy. 2020;13:27-34. doi:10.2147/RMHP.S225388

3. World Health Organization. Depression and Other Common Mental Disorders: Global Health Estimates. Geneva: World Health Organization; 2017.

4. Sozeri-Varma G. Depression in the elderly: clinical features and risk factors. Aging Dis. 2012;3(6):465-471.

5. Bredesen DE, Amos EC, Canick J, et al. Reversal of cognitive decline in Alzheimer's disease. Aging. 2016;8:1250-1258. doi:10.18632/ aging. 100981

6. Cremers G, Taylor E, Hodge L, et al. Effectiveness and acceptability of low-intensity psychological interventions on the well-being of older adults: a systematic review. Clin Gerontol. 2019;11:1-21. doi:10.1080/ 07317115.2019.1662867

7. Bordet R, Ihl R, Korczyn AD, et al. Towards the concept of disease-modifier in post-stroke or vascular cognitive impairment: a consensus report. BMC Med. 2017;15(1):107. doi:10.1186/s12916017-0869-6

8. Cantone M, Bramanti A, Lanza G, et al. Cortical plasticity in depression. ASN Neuro. 2017;9(3):1759091417711512. doi:10.1177/ 1759091417711512
9. Lanza G, Centonze SS, Destro G, et al. Shiatsu as an adjuvant therapy for depression in patients with Alzheimer's disease: a pilot study. Complement Ther Med. 2018;38:74-78. doi:10.1016/j.ctim.20 18.04 .013

10. Vinciguerra L, Lanza G, Puglisi V, et al. Update on the neurobiology of vascular cognitive impairment: from lab to clinic. Int J Mol Sci. 2020;21(8):2977. doi:10.3390/ijms21082977

11. Wilberforce M, Batten E, Challis D, et al. The patient experience in community mental health services for older people: a concept mapping approach to support the development of a new quality measure. BMC Health Serv Res. 2018;18(1):461. doi:10.1186/s12913-0183231-6

12. Rubinstein LZ, Rubinstein LV. Multidimensional geriatric assessment. In: Fillit H, Rockwood K, Young JB, editors. Brocklehurst's Textbook of Geriatric Medicine and Gerontology. 8th edn. Elsevier Science Limited; 2016.

13. Youn JH, Ryu SH, Lee JY, et al. Brain structural changes after multi-strategic metamemory training in older adults with subjective memory complaints: a randomized controlled trial. Brain Behav. 2019;9(5):e01278. doi:10.1002/brb3.1278

14. Woods DL, Kishiyamaa MM, Lund EW, et al. Improving digit span assessment of short-term verbal memory. J Clin Exp Neuropsychol. 2011;33(1):101-111. doi:10.1080/13803395.2010.493149

15. Ji L, Pearlson GD, Zhang X, et al. Physical exercise increases involvement of motor networks as a compensatory mechanism during a cognitively challenging task. Int $J$ Geriatr Psychiatry. 2018;33 (8):1153-1159. doi:10.1002/gps.4909

16. Bennett DA, Schneider JA, Tang Y, Arnold SE, Wilson RS. The effect of social networks on the relation between Alzheimer's disease pathology and level of cognitive function in old people: a longitudinal cohort study. Lancet Neurol. 2006;5(5):406-412. doi:10.1016/S1474-4422(06)70417-3

17. Kuiper JS, Zuidersma M, Oude Voshaar RC, et al. Social relationships and risk of dementia: a systematic review and meta-analysis of longitudinal cohort studies. Ageing Res Rev. 2015;22:39-57. doi:10.1016/j.arr.2015.04.006

18. Glei DA, Landau DA, Goldman N, et al. Participating in social activities helps preserve cognitive function: an analysis of a longitudinal, population-based study of the elderly. Int $J$ Epidemiol. 2005;34(4):864-871. doi:10.1093/ije/dyi049

19. Martin P, Kelly N, Kahana B, et al. Defining successful aging: a tangible or elusive concept? Gerontologist. 2015;55(1):14-25. doi:10.1093/geront/gnu044

20. Park M, Unützer J. Geriatric depression in primary care. Psychiatr Clin North Am. 2011;34:469-487. doi:10.1016/j.psc.2011.02.009

21. Blair AC, Bird MJ. A pilot trial of psychological therapy groups for the very old in residential care: clinical and logistical issues. Clin Psychol. 2016;20(2):68-79. doi:10.1111/cp.12031

22. Chan P, Bhar S, Davison TE. Characteristics of cognitive behavioral therapy for older adults living in residential care: protocol for a systematic review. J Med Internet Res. 2018;20(7):e164.

23. Morga P, Traczyk J, Wittenbeck K, et al. A Review of research on the impact of physical activity on mental state of the elderly. Fizjoterapia. 2015;23(2):42-51.

24. Padberg I, Hotter B, Liebenau A, et al. Unmet need for social and emotional support and lack of recalled screening is associated with depression in the long-term course after stroke. Risk Manag Healthc Policy. 2020;13:285-293. doi:10.2147/RMHP.S228265

25. Cholewa J, Gorzkowska A, Kunicki M, et al. Continuation of full time employment as an inhibiting factor in Parkinson's disease symptoms. Work. 2016;54(3):569-575. doi:10.3233/WOR-162305

26. Nawrocka A, Garbaciak W, Cholewa J, et al. The relationship between meeting of recommendations on physical activity for health and perceived work ability among white-collar workers. Eur J Sport Sci. 2018;18(3):415-422. doi:10.1080/17461391.2018. 1424257 
27. Hirosaki M, Ohira T, Kajiura M, et al. The impact of a programme of exercise and laughter therapy on physical and mental health on elderly persons living in Japan; studying programme effects. Geriatr Gerontol Int. 2013;13(1):152-160. doi:10.1111/j.14470594.2012.00877.x

28. Eyigor S, Karapolat H, Durmaz B, et al. Determining whether a Turkish folk dance has an impact on physical performance, balance, depression and quality of life in elderly women. Arch Gerontol Geriatr. 2009;48(1):84-88. doi:10.1016/j.archger.2007.10.008

29. Pennisi M, Lanza G, Cantone M, et al. Correlation between motor cortex excitability changes and cognitive impairment in vascular depression: pathophysiological insights from a longitudinal TMS study. Neural Plast. 2016;2016:8154969. doi:10.1155/2016/8154969
30. Bella R, Pennisi G, Cantone M, et al. Clinical presentation and outcome of geriatric depression in subcortical ischemic vascular disease. Gerontology. 2010;56(3):298-302. doi:10.1159/000272003

31. Perini G, Ramusino MC, Sinforiani E, et al. Cognitive impairment in depression: recent advances and novel treatments. Neuropsychiatr Dis Treat. 2019;15:1249-1258. doi:10.2147/NDT.S199746

32. Slam RW, Kennedy SH, McIntyre RS, et al. Cognitive dysfunction in major depressive disorder: effects on psychosocial functioning and implications for treatment. Can J Psychiatry. 2014;59(12):649-654. doi:10.1177/070674371405901206
Risk Management and Healthcare Policy

\section{Publish your work in this journal}

Risk Management and Healthcare Policy is an international, peerreviewed, open access journal focusing on all aspects of public health, policy, and preventative measures to promote good health and improve morbidity and mortality in the population. The journal welcomes submitted papers covering original research, basic science, clinical \& epidemiological studies, reviews and evaluations,
Dovepress

guidelines, expert opinion and commentary, case reports and extended reports. The manuscript management system is completely online and includes a very quick and fair peer-review system, which is all easy to use. Visit http://www.dovepress.com/testimonials.php to read real quotes from published authors. 